\title{
Diode-pumped 13 W Yb:KGW femtosecond laser
}

\author{
Jinfang Yang (杨金芳) ${ }^{1,2}$ ，Zhaohua Wang (王兆华 $)^{2,5}$ ，Jiajun Song (宋贾俊 $)^{2,3}$, Xianzhi Wang (王羡之) $)^{2,3}$ ， \\ Renchong Lü (吕仁冲) $)^{1,2}$, Jiangfeng Zhu (朱江峰) $)^{1}$, and Zhiyi Wei (魏志义) ${ }^{2,3,4^{*}}$ \\ ${ }^{1}$ School of Physics and Optoelectronic Engineering, Xidian University, Xi'an 710071, China \\ 2 Beijing National Laboratory for Condensed Matter Physics, Institute of Physics, Chinese Academy of Sciences, Beijing 100190, China \\ 3 University of Chinese Academy of Sciences, Beijing 100049, China \\ ${ }^{4}$ Songshan Lake Materials Laboratory, Dongguan 523808, China \\ ${ }^{5}$ CAS Center for Excellence in Ultra-intense Laser Science, Shanghai 201800, China
}

*Corresponding author: zywei@iphy.ac.cn

Received August 2, 2021 | Accepted August 31, 2021 | Posted Online October 14, 2021

\begin{abstract}
We report on a high-power diode-pumped $\mathrm{Yb}: \mathrm{KG}\left(\mathrm{WO}_{4}\right)_{2}$ (Yb:KGW) mode-locked laser with a semiconductor saturable absorber mirror (SESAM]. For $32.7 \mathrm{~W}$ of incident pump power, we generate $261 \mathrm{fs}$ pulses with the maximum average output power of up to $13.0 \mathrm{~W}$ and spectrum centered around $1039 \mathrm{~nm}$ at $68.4 \mathrm{MHz}$, corresponding to $190 \mathrm{~nJ}$ of single pulse energy and $0.72 \mathrm{MW}$ of peak power. The optical-to-optical conversion efficiency is $39.8 \%$, and the slope efficiency is $64.4 \%$. The $\mathrm{Yb}: \mathrm{KGW}$ laser exhibits a power stability better than $0.543 \%$ of the root-mean-square in $2 \mathrm{~h}$.
\end{abstract}

Keywords: ultrafast laser; high-power femtosecond laser; Yb:KGW crystal; semiconductor saturable absorber mirror. DOI: 10.3788/COL202220.021404

\section{Introduction}

In recent years, more efforts have been devoted to the research of ultrashort pulses with high average power for various applications such as pumping of optical parametric oscillators, material micromachining, multi-photon imaging, and terahertz applications ${ }^{[1-3]}$. At present, the Ti:sapphire laser is limited to output power below $4 \mathrm{~W}$ due to the lower pump power and the quantum efficiency of the crystal. In addition, its pump source is quite expensive. In contrast, the $\mathrm{Yb}^{3+}$-doped femtosecond laser has more great potential because it can be pumped by tens or even hundreds of watts laser diodes (LDs), and the center wavelength ranges from $0.9 \mu \mathrm{m}$ to $1.1 \mu \mathrm{m}$. Despite there being many reports on Yb-doped lasers, the trade-off between the average power and pulse duration was still a challenging topic. Therefore, it is very crucial to choose a novel gain medium to meet a wide spectrum supporting the generation of shorter pulses and good thermo-mechanical properties allowing for high-power laser pumping.

So far, many $\mathrm{Yb}$-doped gain media, such as $\mathrm{Yb}: \mathrm{CaF}_{2}$, $\mathrm{Yb}: \mathrm{Y}_{3} \mathrm{Al}_{5} \mathrm{O}_{12}$ (Yb:YAG), $\mathrm{Yb}: \mathrm{Ca}_{4}(\mathrm{Gd}, \mathrm{Y}) \mathrm{O}\left(\mathrm{BO}_{3}\right)_{3}(\mathrm{Yb}: \mathrm{GdYCOB})$, $\mathrm{Yb}: \mathrm{KY}\left(\mathrm{WO}_{4}\right)_{2} \quad(\mathrm{Yb}: \mathrm{KYW}), \quad \mathrm{Yb}: \mathrm{KG}\left(\mathrm{WO}_{4}\right)_{2} \quad$ (Yb:KGW), $\mathrm{Yb}$ : $\mathrm{CaGdAlO}_{4}$ (Yb:CALGO), and Yb:CaYAlO 4 (Yb:CALYO), have proved to be suitable for mode-locked lasers and exhibited good laser performance ${ }^{[4-10]}$. Among these crystals, the Yb:KGW, Yb:CALGO, and Yb:CALYO crystals have the most potential as gain media, which are suitable for generating high-power lasers with short pulse duration ${ }^{[11-15]}$. Using a
Yb:CALGO crystal, 96 fs pulses were obtained with average power of $12.5 \mathrm{~W}$, which is the highest output power from sub-100-fs Yb-doped oscillators ${ }^{[9]}$. In 2021, a Yb:CALGO oscillator delivering $17.8 \mathrm{fs}$ pulses with $26 \mathrm{~mW}$ of average power was reported $^{[16]}$. Moreover, Tian et al. of Xidian University reported on the $98 \mathrm{fs} \mathrm{Yb}$ :CALYO oscillator with $10 \mathrm{~W}$ of average output power at $81 \mathrm{MHz}$ based on Kerr-lens mode-locking ${ }^{[17]}$. Also, much research is focused on the Yb:KGW crystal, which offers wide emission spectrum across $1023 \mathrm{~nm}-1060 \mathrm{~nm}$ and high thermal conductivity with $3.3 \mathrm{~W} \cdot \mathrm{m}^{-1} \cdot \mathrm{K}^{-1}{ }^{[18]}$.

At present, the most common methods to obtain ultrafast lasers are the Kerr-lens and semiconductor saturable absorber mirror (SESAM) mode-locking for Yb-doped oscillators. Kerrlens mode-locking is usually accompanied by other effects such as self-phase modulation, which can increase new spectral components to support shorter pulse duration. In 2017, the Kerrlens mode-locked Yb:KGW laser generated $120 \mathrm{fs}$ pulses at $1.2 \mathrm{~W}$ and $240 \mathrm{fs}$ pulses with the maximum average output power of $2.3 \mathrm{~W}$ due to the limitation of the strong continuous wave (CW) spectrum components ${ }^{[7]}$. Later, the Yb:KGW oscillator generated 78 fs pulses with $0.65 \mathrm{MW}$ of peak power and $50 \mathrm{~nJ}$ of single pulse energy at the repetition rate of $36 \mathrm{MHz}^{[19]}$. In 2019, Meng et al. reported 73 fs pulses with $4 \mathrm{~W}$ at the repetition frequency of $75.5 \mathrm{MHz}^{[20]}$. Furthermore, a Kerr-lens mode-locking initiated with a SESAM Yb:KGW laser delivered $67 \mathrm{fs}$ pulses with $3 \mathrm{~W}$ of average output power ${ }^{[21]}$. Recently, pulses as short as 56 fs were demonstrated at an 
average power of $1.95 \mathrm{~W}$, which is the shortest pulse duration from a Yb:KGW laser ${ }^{[22]}$. However, the reports mentioned above indicated that increasing output power to $10 \mathrm{~W}$ is challenging with Kerr-lens mode-locking due to small spot size on a crystal. In contrast, pure SESAM mode-locking has more potential in scaling output power due to flexible parameters, such as modulation depth, saturation energy density, and others. The most noteworthy advantage is that it has high stability and self-start capability. Moreover, the cavity adjustment makes it simple to obtain a high-power laser with suitable power density on the SESAM. In 2000, Brunner et al. reported the first, to the best of our knowledge, SESAM mode-locked Yb:KGW laser, where $176 \mathrm{fs}$ pulses with an average power of $1.1 \mathrm{~W}$ were obtained $^{[23]}$. Then, $100 \mathrm{fs}$ pulses have been demonstrated with $126 \mathrm{~mW}$ of output power based on the $\mathrm{Yb}: \mathrm{KGW}$ crystal $^{[24]}$. Furthermore, the $\mathrm{Yb}: \mathrm{KGW}$ oscillator generated 350 fs pulses with $2.4 \mathrm{~W}$ of output power ${ }^{[14]}$. In 2008, Calendron of high-Q laser production Gmbh et al. demonstrated $24 \mathrm{~W}$ CW laser using the dual Yb:KYW crystals and two LDs. Using SESAM, an average output power of $17 \mathrm{~W}$ was obtained in the positive dispersion regime. The pulse duration was around $450 \mathrm{fs}^{[25]}$. Compared to the single crystal, dual crystals can make the small signal gain larger and improve the optical-to-optical conversion efficiency. However, this makes the resonator design complicated and adds the cost of the laser system. In 2015, Kisel et al. demonstrated a diode-pumped SESAM mode-locked $\mathrm{Yb}$ :KGW laser, where the average output power was $8.8 \mathrm{~W}$, and the pulse duration was $162 \mathrm{fs}^{[26]}$. In 2019, we demonstrated a diode-pumped SESAM mode-locked Yb:KGW oscillator, which delivered $160 \mathrm{fs}$ pulse with $7.6 \mathrm{~W}$ of average output power ${ }^{[21]}$. The Yb:KGW crystal can be used not only to generate highpower laser but also to obtain large energy laser ${ }^{[27,28]}$. In 2020, the Yb:KGW regenerative amplifier delivering $227 \mathrm{fs}$ pulses with single pulse energy of $1.2 \mathrm{~mJ}$ at the repetition frequency of $1 \mathrm{kHz}$ after compression was reported ${ }^{[28]}$. Thus, it can be seen that the higher average power is usually achieved at the cost of a longer pulse.

In this work, the single LD was used as the pump source, and one $\mathrm{Yb}: \mathrm{KGW}$ crystal was used as the gain medium. We demonstrate a high-power, SESAM mode-locked Yb:KGW oscillator delivering $261 \mathrm{fs}$ pulses with $13.0 \mathrm{~W}$ of average output power at the repetition rate of $68.4 \mathrm{MHz}$, corresponding to $190 \mathrm{~nJ}$ of single pulse energy and $0.72 \mathrm{MW}$ of peak power. The power stability root-mean-square (RMS) for $2 \mathrm{~h}$ was less than $0.543 \%$, and the laser exhibited a high beam quality.

\section{Experiments}

The schematic experimental configuration of the SESAM modelocked Yb:KGW laser is shown in Fig. 1. An unpolarized multimode fiber coupled LD with $105 \mu \mathrm{m}$ of fiber core diameter and 0.22 of numerical aperture was used as pump source, where its emission wavelength was $980 \mathrm{~nm}$, and the maximum output power was $50 \mathrm{~W}$. An Nm-cut 3\%-doped Yb:KGW crystal (CASTECH, China) was used as gain material, and its size

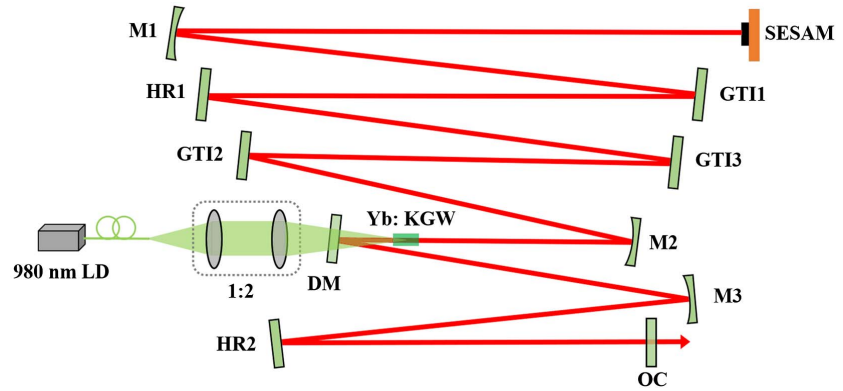

Fig. 1. Experimental configuration of the SESAM mode-locked Yb:KGW laser. $\mathrm{LD}$, laser diode; $\mathrm{Yb}: K G W, \mathrm{Yb}: K G\left(\mathrm{WO}_{4}\right)_{2} ; \mathrm{SESAM}$, semiconductor saturable absorber mirror; DM, dichroic mirror; HR, high reflection mirror; M1, M2, M3, concave mirrors $[R=500 \mathrm{~mm}, 300 \mathrm{~mm}, 500 \mathrm{~mm}$, respectively); OC, output coupler; GTI, Gires-Tournois interferometer mirror.

was $5 \mathrm{~mm}$ (length) $\times 3 \mathrm{~mm}$ (width) $\times 3 \mathrm{~mm}$ (height). In addition, the crystal was antireflection-coated around $980 \mathrm{~nm}-$ $1100 \mathrm{~nm}$. In order to reduce the heat accumulated inside the crystal with high-power pumping, the crystal was mounted on a copper sink and cooled to $14^{\circ} \mathrm{C}$. The crystal has a single pass pump absorption rate of $70 \%-80 \%$, depending on the pump power. The pump laser was focused into the crystal by a coupling system with a magnification of 2.0. The waist radius was $100 \mu \mathrm{m}$ in the center of the Yb:KGW crystal. In order to obtain Fourier limited pulse width, we used three GiresTournois interferometer mirrors (GTI1, GTI2, and GTI3) to control intracavity dispersion, with a total group velocity dispersion (GVD) of $-5800 \mathrm{fs}^{2}$. The "DM" was a dichroic mirror coated for high reflection (HR) across 1030-1200 nm and high transmission (HT) across 820-990 nm. The "M1," "M2," and "M3" represent the concave mirrors coated for HR $(R>99.9 \%)$ across $1000-1065 \mathrm{~nm}$, and the radius of curvature was $500 \mathrm{~mm}, 300 \mathrm{~mm}$, and $500 \mathrm{~mm}$, respectively. Both "HR1" and "HR2" were plane mirrors with fused silica substrates coated for HR in the range of 1000-1065 nm. The output coupler (OC) mirror with a transmittance of $10 \%$ was used in the resonator. All of the laser-coated cavity mirrors used in the experiment, including the HR mirrors, DMs, and OCs (Layertec GmbH, Germany), were designed to have lower dispersion and higher reflectivity to obtain short pulse duration and reduce transmission loss. In order to obtain soliton mode-locking, the SESAM with high optical quality was inserted into the cavity to initiate and maintain mode-locking. As a semiconductor material, the SESAM is vulnerable to heat damage. Therefore, it was mounted on a water-cooled copper heat sink and cooled to $14^{\circ} \mathrm{C}$. The parameters of the SESAM were as follows: modulation depth of $2.4 \%$, relaxation time of $1 \mathrm{ps}$, and saturation fluence of $F_{\text {sat }}=$ $70 \mu \mathrm{J} / \mathrm{cm}^{2}$ at the center of $1064 \mathrm{~nm}$. The SESAM was placed on the translation stages to control the distance between the SESAM and M1. The distance determines the cavity mode size and power density on SESAM and has effects on scaling the output power and the mode-locking stability. Both M2 and M3 were also fixed on the precise translation stages to control the spot distribution in the resonator and optimize the mode matching 
between the pump and the laser. The length of the resonator is $2174 \mathrm{~mm}$, corresponding to the repetition frequency of $69 \mathrm{MHz}$.

\section{Results and Discussion}

In this experiment, we obtained the CW mode-locked laser using the SESAM as an end mirror to initiate and maintain the mode-locking. Firstly, with $8 \%$ transmittance output couple mirror and GTI mirrors of a total GVD of $-4800 \mathrm{fs}^{2}$, we obtained 196 fs pulses with $8.3 \mathrm{~W}$ of average output power. We focus on scaling output power, so the lower transmission OCs were not used. Then, using the $10 \%$ transmission output couple mirror, we measured the average output power, pulse duration, spectral bandwidth, radio frequency (RF) spectrum, beam quality factor, and so on. In a linear cavity, the relationship between output power and pump power was measured, as

(a)

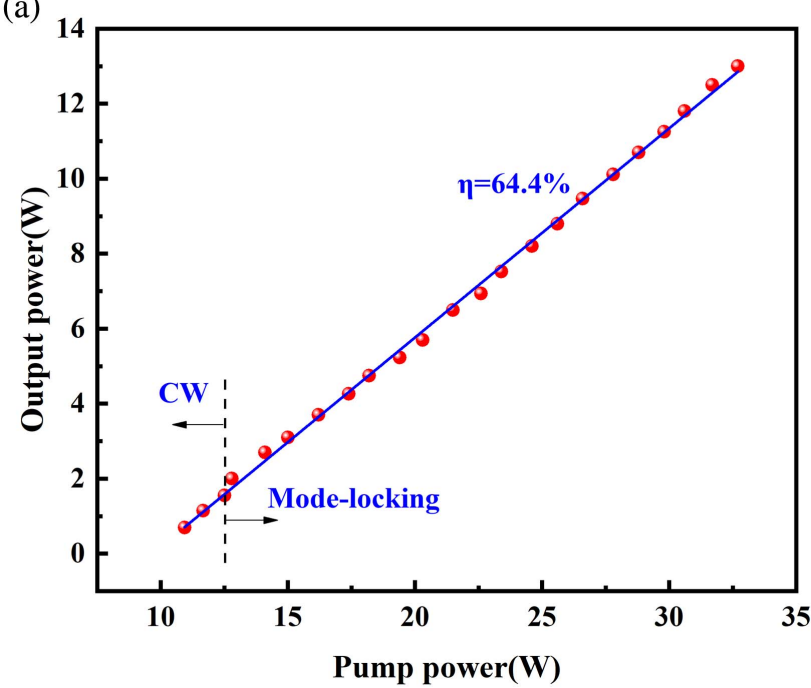

(b)

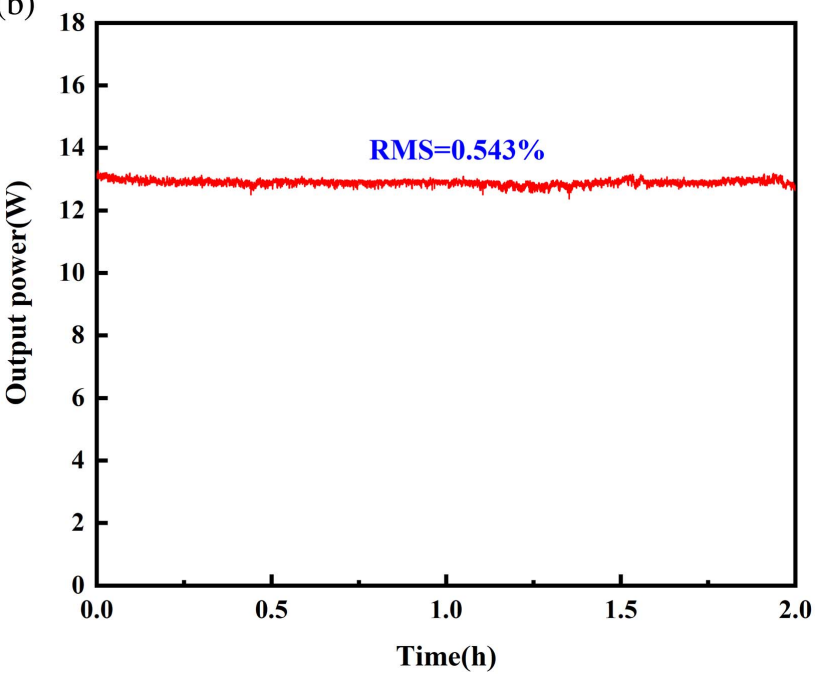

Fig. 2. (a) Output power as a function of the pump power. The blue solid line has a slope efficiency of $64.4 \%$. (b) Power fluctuations at $13 \mathrm{~W}$ average output power in $2 \mathrm{~h}$. shown in Fig. 2(a). It was seen that the output power suddenly increases faster at the pump power of $12.5 \mathrm{~W}$. It means that the pump power threshold of the mode-locked state was $12.5 \mathrm{~W}$. With the pump power of $32.7 \mathrm{~W}$, the maximum average output power operating in the SESAM mode-locked regime was $13 \mathrm{~W}$, corresponding to a slope efficiency of $64.4 \%$ and the optical-tooptical conversion efficiency of $39.8 \%$. Compared to $15.0 \mathrm{~W}$ of $\mathrm{CW}$ power, the output power operating in the mode-locking region dropped mainly due to the transmission loss introduced by GTI mirrors and the unsaturated loss of the SESAM. It is worth mentioning that the appropriate distance between the SESAM and M1 concave mirror is vital to scale the output power. When the distance was between $252 \mathrm{~mm}$ and $258 \mathrm{~mm}$, the mode-locked laser ran in a good state. The spot radius on the SESAM was $180 \mu \mathrm{m}$ in this experiment. Figure 2(b) shows the

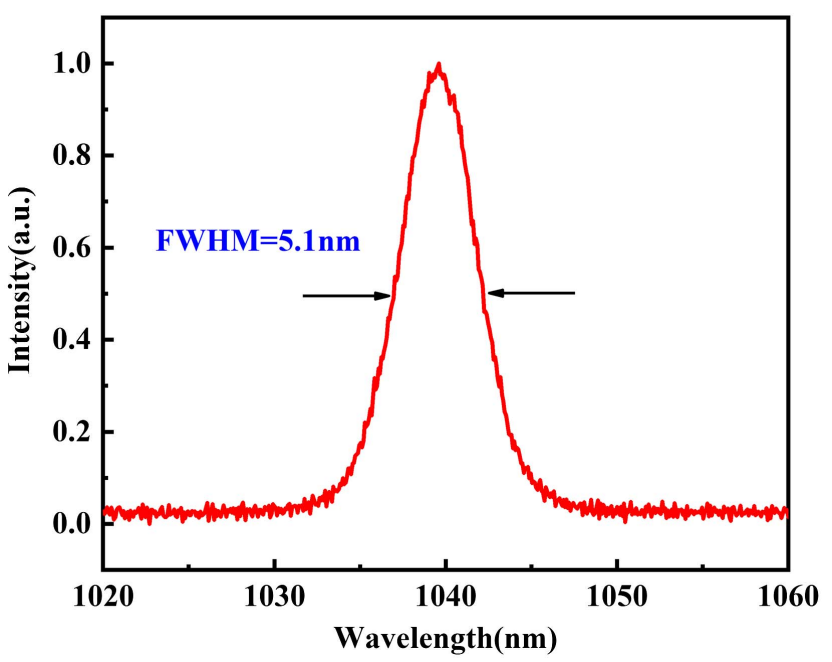

Fig. 3. Spectral distribution of Yb:KGW mode-locked laser at the average output power of $13 \mathrm{~W}$.

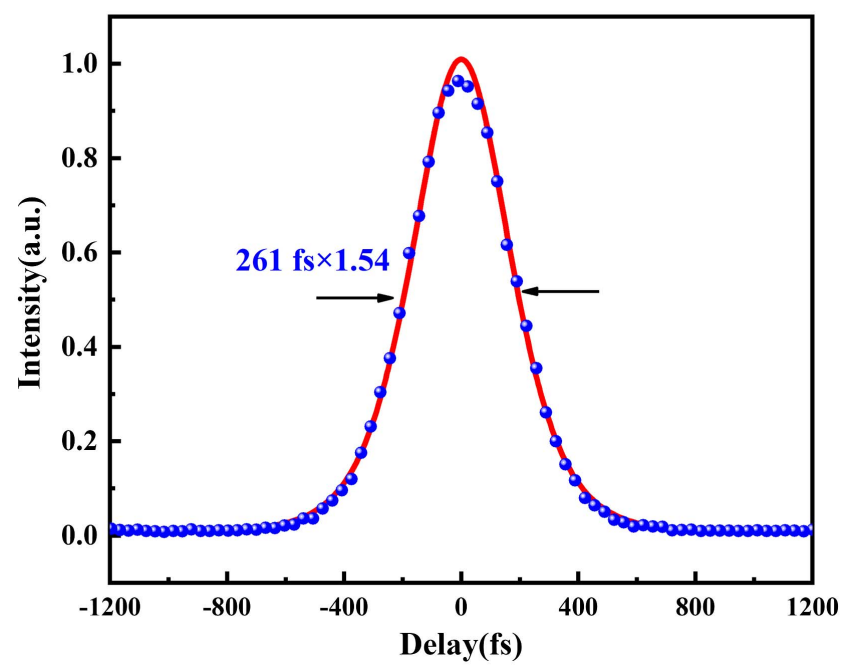

Fig. 4. Measured intensity autocorrelation trace of $\mathrm{Yb}: \mathrm{KGW}$ femtosecond laser at the central wavelength of $1039 \mathrm{~nm}$. 


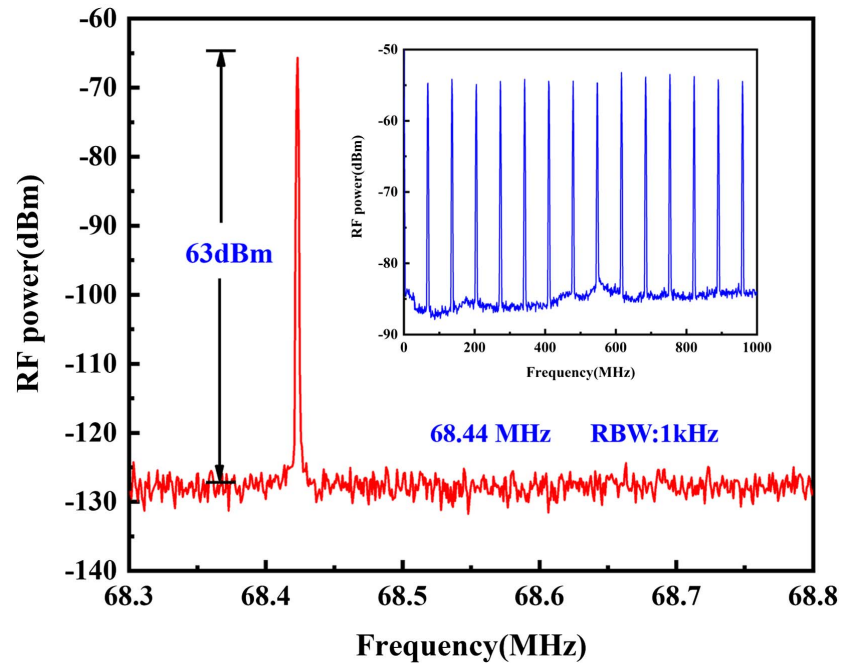

Fig. 5. Typical RF with an RBW of $1 \mathrm{kHz}$. Inset: RF spectrum at $1 \mathrm{GHz}$ wide span with the RBW of $200 \mathrm{kHz}$.

power fluctuations at $13 \mathrm{~W}$ of average power, and the RMS stability was about $0.543 \%$ in $2 \mathrm{~h}$; this exhibited the high performance of the $\mathrm{Yb}: \mathrm{KGW}$ laser. When the pump power was greater than $32.7 \mathrm{~W}$, both the SESAM and the gain crystal were easily damaged. Even if given perturbation, the mode-locking still cannot start again.

As released in Fig. 3, the optical spectrum was measured by an optical spectrum analyzer (OSA, Yokogawa AQ6370C) and centered at $1039 \mathrm{~nm}$ with a full width at half-maximum (FWHM) spectral bandwidth of $5.1 \mathrm{~nm}$, corresponding to $222 \mathrm{fs}$ of Fourier limited pulse duration. With a total negative group delay dispersion (GDD) of $5800 \mathrm{fs}^{2}$, as short as $261 \mathrm{fs}$ pulses assuming $\mathrm{sech}^{2}$ fitting were obtained at maximum output power of $13 \mathrm{~W}$, as shown in Fig. 4.

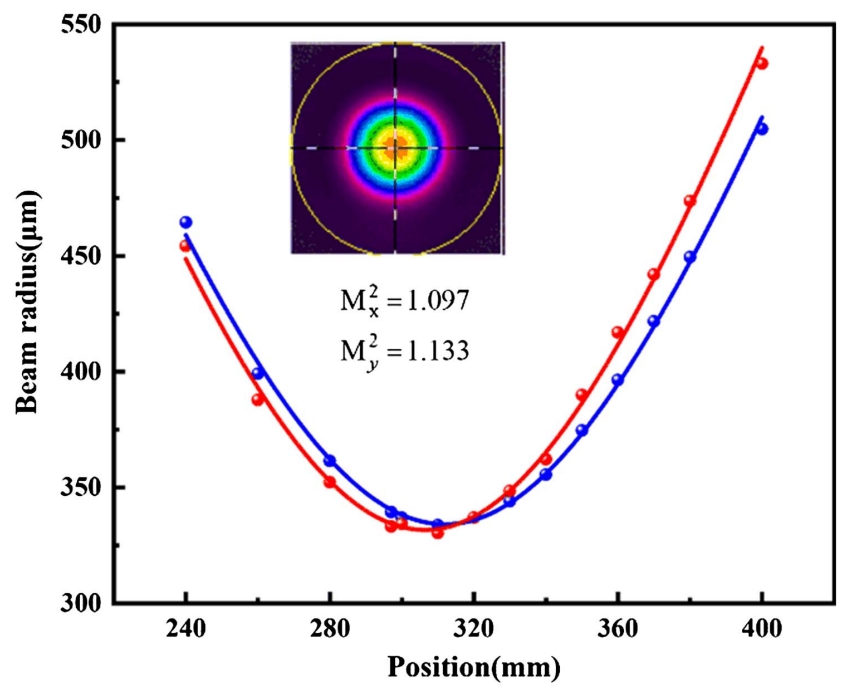

Fig. 6. Measured beam quality factor $M^{2}$ of $\mathrm{Yb}: K G W$ femtosecond oscillator at the $13 \mathrm{~W}$ average output power.
As can be seen from the RF spectrum with a resolution bandwidth (RBW) of $1 \mathrm{kHz}$ displayed in Fig. 5, the fundamental tone at $68.44 \mathrm{MHz}$ measured by an RF spectrum analyzer (E4402B, Agilent) was more than $63 \mathrm{~dB}$ above the noise floor. With an RBW of $200 \mathrm{kHz}$, an inset in Fig. 5 shows that no additional peaks between the higher-order modes were observed. The beam quality factors were measured by a commercial $M^{2}$ factor meter (Spiricon $\mathrm{M}^{2}$-200s), as described in Fig. 6. At $13 \mathrm{~W}$ of maximum output power, the beam quality factors were $M_{x}^{2}=1.097$ and $M_{y}^{2}=1.133$ in the horizontal and vertical directions, respectively. These indicated that the mode-locking oscillator exhibited a high laser performance.

\section{Conclusion}

In conclusion, we have reported on a high-power diode-pumped SESAM mode-locked Yb:KGW laser. With the $10 \%$ transmission OC, the maximum average output power directly generated from the oscillator was up to $13.0 \mathrm{~W}$, corresponding to the optical-to-optical conversion efficiency of $39.8 \%$. When the total GVD was $-5800 \mathrm{fs}^{2}$, the oscillator delivered $261 \mathrm{fs}$ pulses centered around $1039 \mathrm{~nm}$ with peak power of $0.72 \mathrm{MW}$ and per pulse energy of $190 \mathrm{~nJ}$ at a repetition rate of $68.4 \mathrm{MHz}$.

In order to scale the output power while persisting short pulse duration in the SESAM mode-locked regime, some improvements as follows could be made. Firstly, increasing the radius of the spot on the crystal can improve the utilization rate of the pump laser and allow high-power laser pumping. Meanwhile, the thin fused quartz is inserted into the resonator to introduce self-phase modulation resulting in a broader spectrum, and more precise dispersion is also necessary to obtain shorter pulse duration. Finally, the SESAM with high optical quality and high damage threshold is used to optimize the output laser parameters. This will be a potential laser source in various applications.

\section{Acknowledgement}

This work was supported by the National Natural Science Foundation of China (No. 11774410) and Strategic Priority Research Program of CAS (No. XDB16030200).

\section{Reference}

1. J. Song, X. Meng, Z. Wang, X. Wang, W. Tian, J. Zhu, S. Fang, H. Teng, and Z. Wei, "Harmonically pump a femtosecond optical parametric oscillator to $1.13 \mathrm{GHz}$ by a femtosecond $515 \mathrm{~nm}$ laser," Chin. Opt. Lett. 18, 033201 (2020).

2. C. F. O'Donnell, S. Chaitanya Kumar, P. G. Schunemann, and M. EbrahimZadeh, "Femtosecond optical parametric oscillator continuously tunable across $3.6-8 \mu \mathrm{m}$ based on orientation-patterned gallium phosphide," Opt. Lett. 44, 4570 (2019).

3. K. Ahmmed, C. Grambow, and A.-M. Kietzig, "Fabrication of micro/nano structures on metals by femtosecond laser micromachining," Micromachines 5, 1219 (2014).

4. G. Machinet, P. Sevillano, F. Guichard, R. Dubrasquet, P. Camy, J. L. Doualan, R. Moncorge, P. Georges, F. Druon, D. Descamps, and 
E. Cormier, "High-brightness fiber laser-pumped 68 fs $2.3 \mathrm{~W}$ Kerr-lens mode-locked $\mathrm{Yb}: \mathrm{CaF}_{2}$ oscillator," Opt. Lett. 38, 4008 (2013).

5. G. Ju, L. Chai, Q. Wang, Z. Zhang, Y. Wang, and X. Ma, "Stable mode-locking in an Yb: YAG laser with a fast SESAM," Chin. Opt. Lett. 1, 695 (2003).

6. H. Zeng, H. Lin, Z. Lin, L. Zhang, Z. Lin, G. Zhang, V. Petrov, P. Loiko, X. Mateos, L. Wang, and W. Chen, "Diode-pumped sub-50 fs Kerr-lens mode-locked Yb:GdYCOB laser,” Opt. Express 29, 13496 (2021).

7. R. Akbari and A. Major, "High-power diode-pumped Kerr-lens mode-locked bulk Yb:KGW laser," Appl. Opt. 56, 8838 (2017).

8. F. Mejiti, V. L. Kalashnikov, I. G. Poloyko, and T. Vajidi, "Kerr lens modelocked operation of Yb: KYW laser," Chin. J. Lasers B 11, 166 (2002).

9. A. Greborio, A. Guandalini, and J. Aus der Au, "Sub-100 fs pulses with 12.5-W from Yb:CALGO based oscillators," in Proc. SPIE 8235, 823511 (2012).

10. W. Tian, C. Yu, J. Zhu, D. Zhang, Z. Wei, X. Xu, and J. Xu, "Diode-pumped high-power sub-100 fs Kerr-lens mode-locked $\mathrm{Yb}: \mathrm{CaYAlO}_{4}$ laser with 1.85 MW peak power," Opt. Express 27, 21448 (2019).

11. B. Lee, B. Jeong, J. W. Kim, E. G. Sall, C. Kim, S. Park, D. Heo, S. A. Chizhov, J. Yang, V. E. Yashin, and G.-H. Kim, "High-power Yb:YAG thin-rod amplifier for use in a regenerative amplifier based on dual-slab Yb:KGW crystals," Laser Phys. 31, 065001 (2021).

12. S. Manjooran and A. Major, "Diode-pumped $45 \mathrm{fs}$ Yb:CALGO laser oscillator with 1.7 MW of peak power," Opt. Lett. 43, 2324 (2018).

13. N. Modsching, C. Paradis, F. Labaye, M. Gaponenko, I. J. Graumann, A. Diebold, F. Emaury, V. J. Wittwer, and T. Sudmeyer, "Kerr lens modelocked Yb:CALGO thin-disk laser,” Opt. Lett. 43, 879 (2018).

14. J. F. Li, X. Y Liang, J. P. He, and H. Lin, "Stable, efficient diode-pumped femtosecond $\mathrm{Yb}$ :KGW laser through optimization of energy density on SESAM," Chin. Opt. Lett. 9, 071406 (2011).

15. L. Zheng, W. Tian, H. Liu, G. Wang, C. Bai, R. Xu, D. Zhang, H. Han, J. Zhu, and Z. Wei, "2-GHz watt-level Kerr-lens mode-locked Yb:KGW laser," Opt. Express 29, 12950 (2021).

16. Y. Wang, X. Su, Y. Xie, F. Gao, S. Kumar, Q. Wang, C. Liu, B. Zhang, B. Zhang, and J. He, "17.8 fs broadband Kerr-lens mode-locked Yb: CALGO oscillator," Opt. Lett. 46, 1892 (2021).
17. W. Tian, R. Xu, L. Zheng, X. Tian, D. Zhang, X. Xu, J. Zhu, J. Xu, and Z. Wei, "10-W-scale Kerr-lens mode-locked Yb:CALYO laser with sub-100-fs pulses," Opt. Lett. 46, 1297 (2021).

18. N. V. Kuleshov, A. A. Lagatsky, A. V. Podlipensky, V. P. Mikhailov, and G. Huber, "Pulsed laser operation of Yb-doped $\mathrm{KY}\left(\mathrm{WO}_{4}\right)_{2}$ and $\mathrm{KGd}\left(\mathrm{WO}_{4}\right)_{2}$," Opt. Lett. 22, 1317 (1997).

19. H. Zhao and A. Major, "Megawatt peak power level sub-100 fs Yb:KGW oscillators," Opt. Express 22, 30425 (2014).

20. X. Meng, C. lv, Q. Liu, X. Zhang, Y. Li, X. Xi, and B. Zhao, "Diode-pumped Yb:KGW laser with 73 fs pulse and $0.72 \mathrm{MW}$ peak power based on Kerr-lens mode locking," Appl. Phys. B 125, 166 (2019).

21. H. Zhao and A. Major, "Powerful $67 \mathrm{fs}$ Kerr-lens mode-locked prismless Yb: KGW oscillator," Opt. Express 21, 31846 (2013).

22. R. Akbari, K. A. Fedorova, E. U. Rafailov, and A. Major, "Diode-pumped ultrafast Yb:KGW laser with 56 fs pulses and multi-100 kW peak power based on SESAM and Kerr-lens mode locking," Appl. Phys. B 123, 123 (2017).

23. F. Brunner, G. J. Spühler, J. Aus der Au, L. Krainer, F. Morier-Genoud, R. Paschotta, N. Lichtenstein, S. Weiss, C. Harder, A. A. Lagatsky, A. Abdolvand, N. V. Kuleshov, and U. Keller, "Diode-pumped femtosecond $\mathrm{Yb}: \mathrm{KGd}\left(\mathrm{WO}_{4}\right)_{2}$ laser with 1.1-W average power," Opt. Lett. 25, 1119 (2000).

24. G. Paunescu, J. Hein, and R. Sauerbrey, "100-fs diode-pumped Yb:KGW mode-locked laser," Appl. Phys. B 79, 555 (2004).

25. A. L. Calendron, K. S. Wentsch, and M. J. Lederer, "High power $\mathrm{cw}$ and mode-locked oscillators based on Yb:KYW multi-crystal resonators," Opt. Express 16, 18838 (2008).

26. V. E. Kisel, A. S. Rudenkov, A. A. Pavlyuk, A. A. Kovalyov, V. V. Preobrazhenskii, M. A. Putyato, N. N. Rubtsova, B. R. Semyagin, and N. V. Kuleshov, "High-power, efficient, semiconductor saturable absorber mode-locked Yb:KGW bulk laser,” Opt. Lett. 40, 2707 (2015).

27. D. Y. Yan, B. W. Liu, Y. X. Chu, H. Y. Song, L. Chai, M. L. Hu, and Q. Y. Wang, "Hybrid femtosecond laser system based on a Yb:KGW regenerative amplifier for NP polarization," Chin. Opt. Lett. 17, 041404 (2019).

28. H. He, J. Yu, W. Zhu, X. Guo, C. Zhou, and S. Ruan, "A Yb:KGW dual-crystal regenerative amplifier," High Power Laser Sci. Eng. 8, e35 (2020). 